\title{
ASCO 2013: from the rediscovery of old recipes to targeted oncology
}

\author{
Jean-François Morère
}

Received: 9 July 2013 / Accepted: 9 July 2013 / Published online: 23 August 2013

(C) Springer-Verlag France 2013

This last American Society of Clinical (ASCO) meeting was really balanced between empirical medicine and precision medicine.

Though both are certainly important, the greatest emotional impact this year must be credited to the use of an old recipe for the detection of cervical cancer-acetic acid, also known as vinegar. When combined with visual inspection, this technique constitutes an old-fashioned but effective test for the early diagnosis of precancerous and invasive lesions. The modern idea is to educate primary health workers to perform this test in underprivileged communities in India. A randomized study on this subject included 150,000 women over a period of 15 years [1].

The subjects received four rounds of screening at 24month intervals. In the control group, subjects were requested to contact the hospital if typical symptoms of cervical cancer should arise, on the basis of what they had learned during an initial educational session.

This seemed to work quite well, with a reduction of $30 \%$ in mortality due to cervical cancer in the screened group. According to Surendra Srinivas Shastri, MD, lead author of the study, "This is the first trial to identify a cervical cancer screening strategy that reduces mortality and is feasible for implementation on a broad scale throughout India and in other developing countries. Our trial used primary health care workers who can easily access women in the community, which is critical in India and other countries that lack sufficient nurses, physicians, and laboratory facilities." Not surprisingly, these researchers received a warm welcome and standing ovation during the plenary session.

On the other hand, the new futuristic recipes used in "targeted oncology" are also receiving attention. However,

J.-F. Morère $(\bowtie)$

Oncology Department, Paul Brousse University Hospital,

Villejuif, France

e-mail: targeted@hotmail.fr the main target appears to have changed somewhat, being no longer the signal transmission pathways but instead immunological determinants.

In the latest years, we already had breaking news with monoclonal antibodies targeting CTLA4 in melanoma. The main results were a high response rate for the majority of patients and, for a few of them, a new concept in melanoma therapeutics - the concept of long survivors [2]. This year, immunotherapy has reinforced its status at the top of the hits.

First, programmed cell death ligand-1 (PDL-1) is a protein that helps cancer cells to escape from immune cells and is the new target of an engineered monoclonal tumor antibody $0 \mathrm{~A}$, which demonstrated impressive tumor shrinkage in a phase 1 study [3]. The observed efficacy was not restricted to melanoma, but progress was also achieved in lung, stomach, and kidney cancer after several previous standard treatments. "We are impressed with the frequency and duration of the responses in these patients with very difficult-to-treat tumors. So far, almost none of the patients that have had tumor shrinkage have progressed," said Roy S. Herbst, MD, PhD, and Ensign Professor of Medicine at Yale Cancer Center.

Overall, $21 \%$ of the patients suffering from lung cancer and melanoma experienced significant tumor shrinkage. Therapy responses are still ongoing, with the majority (26 out of 29) of responders continuing to respond. Researchers are currently investigating with a companion test whether or not PDL-1 has an impact on tumor response.

According to ASCO president-elect A. Hudis, "The fact that this drug was active in such important immune mechanism. Suggests that PDL-1 is part of a group of drugs that can universally or generally activate and direct the immune system, and which will likely take on a growing role in patient care, and it's particularly exciting to see major effects in patients whose cancer has progressed despite all other standard therapies."

Further, a combination of the two immunotherapies appears to be better than each drug given separately in a phase 1 
study. Ipilimumab targets CTLA-4, and nivolumab is directed against PD1.

For ASCO President Sandra Swain, "The further exploration of immunotherapy as a stand-alone therapy without chemotherapy for a devastating disease such as advanced melanoma is a tremendous advance. We are identifying therapies that work better together than alone, and look promising for more than just a small subset of patients. The extensive tumor shrinkage that was observed in this study is exactly the type of benefit patients and doctors have been hoping for."

As we see, immunotherapies targeting PDL-1, PD1, and CTLA- 4 are leading the way in the battle against refractory cancer. There have also been new advances with more "classical" targeted therapies, beginning with ganetespib, a first in its class of new anti-HSP 90 drugs active as a second-line therapy for non-small lung cancer; selumetinib, the first medical treatment for eye melanoma; and pazopanib in the maintenance treatment after chemotherapy of ovarian carcinomas.

Nintedanib, a new antiangiogenic, seems to be promising in combination with chemotherapy in patients with nonsmall cell lung cancer that has evaded previous treatment. Sorafenib can stop tumor growth in differentiated carcinomas of the thyroid that cannot be successfully treated with iodine radiotherapy.

In combination with chemotherapy, bevacizumab represents a new standard treatment with a positive impact on overall survival ( 17 versus 13.3 months median survival) in advanced cervical cancer. "Women with advanced cervical cancer don't have many options. We finally have a drug that helps women live longer," declared the lead study author Krishnansu Sujata Tewari.

With this study, things come full circle, at least for cervical cancer-from old recipes to new ones. Long live the new and innovative recipes in precision medicine!

Conflicts of Interest Research funds, avisory boards: Roche, Pfizer, Astra Zeneca, Teva, Boehringer.

\section{References}

1. Surendra Srinivas Shastri, Indraneel Mittra, Gauravi Mishra, Subhadra Gupta, Rajesh Dikshit, Rajendra A. Badwe (2013) Effect of visual inspection with acetic acid (VIA) screening by primary health workers on cervical cancer mortality: a cluster randomized controlled trial in Mumbai, India. In: 2013 ASCO annual meeting, abstract 2

2. Hodi F, O'DAY S, URBA WJ (2010) Improved survival with ipilimumab in patients with metastatic melanoma. N Engl J Med 19(363):711-723. doi:10.1056/NEJMoa1003466

3. Roy S. Herbst, Michael S. Gordon, Gregg Daniel Fine, Jeffrey Alan Sosman, Jean-Charles Soria, Omid Hamid, John D. Powderly II, Howard A. Burris III, Ahmad Mokatrin, Marcin Kowanetz, Maya Leabman, Maria Anderson, Daniel S. Chen, F. Stephen Hodi (2013) A study of MPDL3280A, an engineered PD-L1 antibody in patients with locally advanced or metastatic tumors. In: 2013 ASCO annual meeting, abstract \#3000 